\title{
Phosphorylated Syntaxin 1 Is Localized to Discrete Domains Along a Subset of Axons
}

\author{
Davide L. Foletti, Richard Lin, Michael A. F. Finley, and Richard H. Scheller \\ Howard Hughes Medical Institute, Department of Molecular and Cellular Physiology, Stanford University School of \\ Medicine, Stanford, California 94305-5428
}

Syntaxin 1 is a SNARE protein that plays a central role in synaptic vesicle (SV) exocytosis. We generated an antibody that specifically recognizes a casein kinase II-mediated phosphorylation on serine-14 of syntaxin 1. In this report we show that this phosphorylation occurs in vivo and is developmentally regulated in the rat brain, rising to a level of $40 \%$ of the total syntaxin in adult animals. Phosphorylated syntaxin is preferen- tially associated with SNAP-25 and localizes to discrete domains of the axonal plasma membrane that do not colocalize with pools of synaptic vesicles. These phosphosyntaxin domains may define fusion sites for a novel class of vesicles outside classical active zones.

Key words: syntaxin 1; phosphorylation; casein kinase II; SNAREs; exocytosis; immunohistochemistry
Regulation of plasma membrane dynamics is critical to the function of the nervous system in many ways. For example, during development, axon and dendrite outgrowth takes place through membrane addition at the growth cone, whereas synapse formation includes the insertion and/or localization of specific proteins at sites of cell contact. In the adult, neurotransmitter release is accomplished through a cycle of vesicle exocytosis and endocytosis. Finally, memory formation likely takes place through the modulation of presynaptic neurotransmitter release and/or postsynaptic receptor organization and requires the intricate regulation of membrane trafficking events.

Crucial steps in the dynamics of the plasmalemma are the addition of new membrane through the process of vesicle fusion and the retrieval of membrane by endocytosis. Several genes or gene families have been implicated in the membrane fusion process and are therefore likely targets for controlling the dynamics of the neuronal plasma membrane. The products of three of these genes, VAMP (also called synaptobrevin), SNAP-25, and syntaxin, are collectively referred to as SNAREs (Südhof, 1995). These proteins form a core fusion complex that is composed of a four-helical bundle spanning the vesicle and target membranes (Poirier et al., 1998a; Sutton et al., 1998). Formation of this complex is a late step in the membrane fusion process and perhaps actually drives fusion of the lipid bilayers (Hanson et al., 1997; Lin and Scheller, 1997).

Given the central role of membrane fusion in the functions of various membrane compartments, it is critical to understand how the SNAREs may be regulated. In particular, insight into the regulation of neuronal SNAREs will most likely be critical in understanding mechanisms of synaptic development and plasticity. The three neuronal SNAREs, VAMP2, syntaxin1, and

\footnotetext{
Received Jan. 27, 1999; revised March 20, 2000; accepted March 27, 2000.

We thank Dr. Chris Kaznowski for assistance in obtaining and analyzing rat brain sections, Dr. Susan Palmieri for assistance with confocal microscopy, and Dr. Susan McConnell for critical reading of this manuscript.

Correspondence should be addressed to Richard H. Scheller, Howard Hughes Medical Institute, Department of Molecular and Cellular Physiology, Stanford University School of Medicine, Stanford, CA 94305-5428. E-mail: scheller@cmgm.stanford.edu.

Copyright (c) 2000 Society for Neuroscience $0270-6474 / 00 / 204535-10 \$ 15.00 / 0$
}

SNAP-25, have been shown to be phosphorylated in vitro by different kinases: VAMP2 by $\mathrm{Ca}^{2+}$ - and calmodulin-dependent protein kinase II (Hirling and Scheller, 1996) and casein kinase II (CKII) (Nielander et al., 1995), syntaxin 1 by casein kinase II (Bennett et al., 1993b; Hirling and Scheller, 1996; Risinger and Bennett, 1999), and SNAP-25 by protein kinase A (Risinger and Bennett, 1999) and protein kinase C (Shimazaki et al., 1996). The in vivo occurrence, functional significance, cell or developmental specificity, and the stimuli that control the phosphorylation of these SNAREs remain largely unknown. In this report we investigate the phosphorylation on serine-14 of syntaxin 1 by casein kinase II using phosphosyntaxin-specific antibodies. We show that this phosphorylation occurs in vivo and that phosphosyntaxin levels increase during development. Specific domains along subsets of axons are marked by phosphosyntaxin, and immunoprecipitation experiments show that phosphorylated syntaxin is enriched in complexes with SNAP-25. Although the phosphosyntaxin occurs in regions of brain that are actively undergoing synaptogenesis, the labeled axonal domains do not correspond to synaptic sites. The data suggest a role for casein kinase II and phosphosyntaxin 1 in defining specific subdomains in the axonal plasma membrane that are segregated from the synaptic active zones. These subdomains are likely enriched in the binary SNAP$25 /$ syntaxin complex and therefore may be primed for the exocytosis of a novel class of vesicles.

\section{MATERIALS AND METHODS}

Antibodies and reagents. The mouse monoclonal antibodies used in this study were anti-MAP2 (Transduction Laboratories, Lexington, KY), anti-tau and anti-synaptophysin (Boehringer Mannheim, Indianapolis, IN), anti-HPC-1 (described in Barnstable et al., 1985), anti-calbindin (Swant, Bellinzona, Switzerland), and anti-SNAP-25 (Sternberger Monoclonals, Lutherville, MD). The affinity-purified polyclonal antiVAMP2 antibody was described previously (Pevsner et al., 1994). The nuclear marker TOTO-3 was purchased from Molecular Probes (Eugene, $\mathrm{OR})$. Secondary antibodies for immunohistochemistry were from Jackson ImmunoResearch Laboratories (West Grove, PA) and included fluorescein isothiocyanate (FITC)-conjugated AffiniPure goat anti-rabbit $\mathrm{IgG}$ and Texas Red (TxR)-conjugated AffiniPure goat anti-mouse IgG. Secondary antibodies for quantitative Western Blot analysis were bought from Amersham Pharmacia Biotech (Arlington, IL) and included antirabbit Ig from donkey, ${ }^{125}$ I-labeled $\mathrm{F}\left(\mathrm{ab}^{\prime}\right)_{2}$ fragment and anti-mouse Ig 
from sheep, ${ }^{125} \mathrm{I}$-labeled $\mathrm{F}\left(\mathrm{ab}^{\prime}\right)_{2}$ fragment. Paraformaldehyde was purchased from Electron Microscopy Sciences (Fort Washington, PA), and casein kinase II (human, recombinant from Escherichia coli) was from Boehringer Mannheim (Indianapolis, IN). Unless stated otherwise, all other reagents were purchased from Sigma (St. Louis, MO) or Fisher Biotech (Pittsburgh, PA).

Generation and purification of $\alpha$ Psyn. A peptide corresponding to amino acids 9-19 of syntaxin 1A (RTAKDSDDDDD) (Bennett et al., 1992) was synthesized with a phosphoserine at position 14 and an additional cysteine residue at the $\mathrm{C}$ terminus (introduced for coupling purposes). The peptide was coupled to Imject maleimide-activated keyhole limpet hemocyanin (KLH) (Pierce, Rockford, IL) and used as immunogen in rabbit. The polyclonal antiserum was affinity-purified as follows. A peptide with unrelated sequence, a peptide with the same sequence but with unphosphorylated serine (related nonphosphopeptide), and the peptide used as immunogen (phosphopeptide) were coupled to Imject maleimide-activated bovine serum albumin (BSA) (Pierce). The conjugated peptides were then linked to cyanogen bromide-activated Sepharose 4B (Sigma). The polyclonal antiserum was first sequentially passed over columns carrying the peptide with unrelated sequence and the related nonphosphopeptide to remove nonspecific antibodies. Finally, the antiserum was affinity-purified by binding and elution from a column carrying the phosphopeptide.

Recombinant proteins and in vitro phosphorylation. The recombinant proteins syn1A11 [rat syntaxin 1A, amino acid (aa) 4-266], SN25N (mouse SNAP-25 N terminus, aa 1-82), SN25C (mouse SNAP-25 C terminus, aa 142-206), and VAMP2 $\Delta 1-24$ (rat VAMP2, aa 25-96) were expressed and purified as described (Yang et al., 1999). SDS-PAGE and Western blotting were performed according to standard protocols. Quantitative Western blots were analyzed by phosphorimaging (Molecular Dynamics, Sunnyvale, CA). The preparation of the SDS-resistant core complex was as described (Yang et al., 1999). In vitro phosphorylation of recombinant syn1A11 was achieved by incubating the protein for $30 \mathrm{~min}$ at $30^{\circ} \mathrm{C}$ in $50 \mathrm{~mm}$ Tris- $\mathrm{HCl}, \mathrm{pH} 7.4,130 \mathrm{~mm} \mathrm{KCl}, 10 \mathrm{~mm} \mathrm{MgCl}_{2}, 1 \mathrm{~mm}$ DTT, $30 \mu \mathrm{M}$ D-sphingosine, $200 \mu \mathrm{M}$ ATP, and $10^{-4} \mathrm{U}$ casein kinase II per 20 pmol of protein. The reaction was stopped by addition of SDSPAGE sample buffer.

Rat brain fractionation and immunoprecipitation. The preparation of rat brain homogenates, postnuclear, membrane, and cytosolic fractions as well as Triton X-100-extracted fractions were as described (Steegmaier et al., 1999). Immunoprecipitation experiments were performed essentially as described (Steegmaier et al., 1999), with the exception that the antibodies were not directly coupled to the protein A or protein G-Sepharose beads (Amersham Pharmacia Biotech); instead, a two-step procedure was used.

Rat brain slices. Rat brain slices were prepared as previously described (McQuinston and Madison, 1999). During preparation, slices were maintained in a high $\mathrm{Mg}^{2+}$, low $\mathrm{Ca}^{2+}(3 \mathrm{~mm}, 1 \mathrm{~mm})$ Ringer's solution containing the glutamate receptor antagonist kynurenic acid (1 $\mathrm{mM})$ to prevent excitotoxicity. Application of activity-modulating drugs was made in normal Ringer's solution $\left(2.5 \mathrm{mM} \mathrm{Ca}^{2+}, 1.3 \mathrm{mM} \mathrm{Mg}^{2+}\right)$ lacking kynurenic acid.

Immunohistochemistry. Postnatal day 5 (P5), P9, and adult rats were anesthetized with an intraperitoneal injection of Nembutal and then sequentially perfused intracardially with ice-cold $0.1 \mathrm{M}$ phosphate buffer and $4 \%$ ice-cold formaldehyde in $0.1 \mathrm{~m}$ phosphate buffer. The brains were then removed from the skull, post-fixed in the same fixative for $2-4 \mathrm{hr}$ on ice, and finally cryoprotected by immersion in $20 \%$ sucrose for $24 \mathrm{hr}$. Frozen brains were cut with a cryostat generating $16-\mu \mathrm{m}$-thick sagittal sections that were mounted on glass slides. The staining protocol was performed at room temperature. The sections were first air-dried for 15 min and then permeabilized for 1-2 hr (this step also served to block unspecific sites) in PBS containing $0.4 \%$ saponin, $1 \%$ BSA, and $2 \%$ normal goat serum (permeabilization/blocking buffer). Primary antibodies diluted in permeabilization/blocking buffer were applied to the sections for $4 \mathrm{hr}$ in a humidified chamber. After the sections were washed five times for $5 \mathrm{~min}$ with permeabilization/blocking buffer, secondary antibodies were applied for 1-2 hr. Finally, the sections were rinsed as above and mounted with Vectashield as mounting medium (Vector Laboratories, Burlingame, CA). Microscopy was performed with a Molecular Dynamics laser confocal imaging system (Stanford University, Cell Sciences Imaging Facility). Additionally, deconvoluted reconstructions of serial sections were obtained by acquiring and processing images with an Olympus IX70 inverted microscope equipped with a CCD camera and Deltavision software (Molecular Dynamics).

\section{RESULTS}

Previous reports (Bennett et al., 1993b; Hirling and Scheller, 1996; Risinger and Bennett, 1999) have shown that syntaxin 1 can be in vitro-phosphorylated by CKII. Immunoprecipitation of synaptotagmin from rat brain lysates resulted in coprecipitation of CKII, and the kinase was shown to phosphorylate synaptotagmin as well as syntaxin 1 (Bennett et al., 1993b). Furthermore, in vitro binding studies have shown that the CKII-mediated phosphorylation of syntaxin 1 enhances its interaction with synaptotagmin (Risinger and Bennett, 1999). In vitro phosphorylation of truncated forms of syntaxin 1 and analysis of its primary structure searching for CKII consensus motifs indicated that serine-14 was a potential phosphorylation site. To elucidate the possible in vivo occurrence, distribution and physiological relevance of a CKIImediated phosphorylation of syntaxin 1 on serine-14, we raised an antibody that selectively recognizes the phosphorylated form of the protein.

\section{The $\alpha$ Psyn antibody is specific for syntaxin 1 phosphorylated on serine-14}

A peptide corresponding to aa 9-19 of syntaxin 1A with a phosphoserine at position 14 was coupled to KLH and used to generate an immune response in rabbit. The resulting polyclonal antiserum was passed over columns carrying an unrelated peptide and the related nonphosphopeptide to remove nonspecific antibodies. Finally, the antiserum was affinity-purified by binding to the phosphorylated peptide. The affinity-purified polyclonal antibody ( $\alpha$ Psyn) proved to be highly specific for the CKIIphosphorylated form of syntaxin 1. In a dot-blot experiment, $\alpha$ Psyn was able to recognize $10 \mathrm{ng}$ of the immunogenic phosphopeptide and did not cross-react with up to $2 \mu \mathrm{g}$ of nonphosphopeptide or with unrelated peptides containing a phosphoserine or phosphothreonine (Fig. 1A). Purified CKII was used to phosphorylate in vitro recombinant syntaxin $1 \mathrm{~A}$ that lacks the membrane anchor (syn1A11). Phosphorylated and unphosphorylated syn1A11 were then resolved side by side by SDS-PAGE, transferred to nitrocellulose, and probed with $\alpha$ Psyn or $\alpha \mathrm{HPC}-1$, a monoclonal antibody directed against syntaxin 1 (Barnstable et al., 1985). Although $\alpha$ HPC-1 detected equally well the phosphorylated and unphosphorylated syn1A11, $\alpha$ Psyn showed selective recognition of the phosphorylated protein (Fig. 1B).

\section{Phosphorylated syntaxin 1A and 1B are found throughout the adult rat brain}

Having established the specificity of $\alpha$ Psyn in recognizing only the phosphorylated form of syntaxin, we probed rat brain fractions for the in vivo presence of phosphosyntaxin. Adult rat brain was homogenized and separated into membrane, cytosolic, and Triton X-100 extracted membrane fractions. The fractions were probed by Western blot with $\alpha \mathrm{HPC}-1$ and $\alpha$ Psyn. As shown previously (Ruiz-Montasell et al., 1996), $\alpha$ HPC-1 recognized a doublet of proteins of $35-38 \mathrm{kDa}$ with the lower band being syntaxin 1A (syn1A) and the upper band corresponding to syntaxin 1B (syn1B) (Fig. 2A, lane 1 ). Similarly, $\alpha$ Psyn recognized in the membrane fraction a doublet of proteins with the same apparent molecular weight as syn1A and syn1B; no proteins were detected in the cytosolic fraction (Fig. 2A, lanes 2 and 3). As expected from the integral membrane protein characteristic of syntaxin 1 , the two proteins recognized by $\alpha$ Psyn are quantitatively extracted from membranes by incubation with Triton X-100 (Fig. 2A, lane 4). No additional bands were detected in these fractions or in the total homogenate fraction (data not shown). 
A

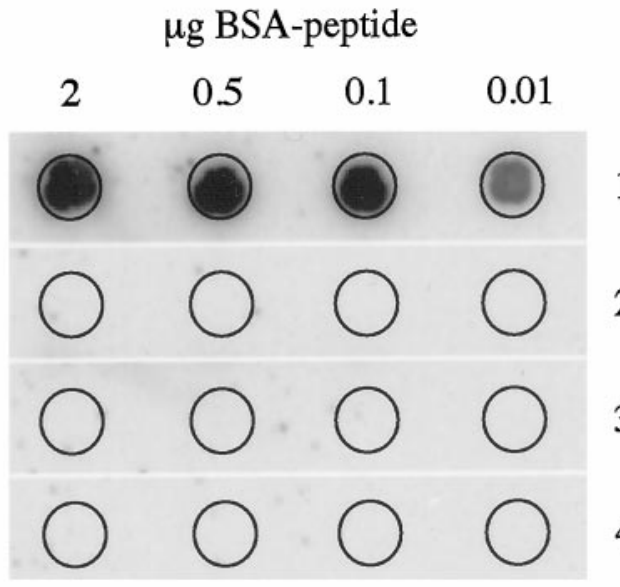

B

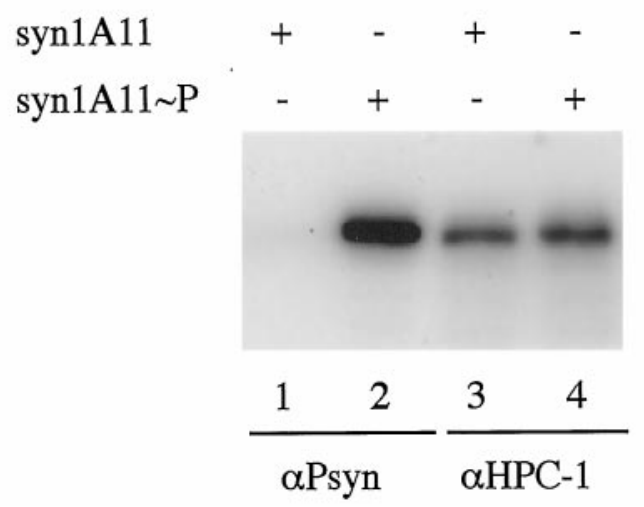

Figure 1. The antibody $\alpha$ Psyn is specific for the serine-14phosphorylated form of syntaxin 1. A, Dot-blot analysis of the specificity of $\alpha$ Psyn. The indicated amounts of peptides coupled to BSA were spotted on a nitrocellulose membrane and probed with the antibody $\alpha$ Psyn. The antibody recognizes only the phosphopeptide that was used as immunogen (1); no cross-reactions are detected against the nonphosphorylated peptide with the same sequence (2), or against unrelated phosphopeptides containing a phosphoserine (3) or a phosphothreonine (4). $B$, Bacterially expressed syntaxin 1 lacking the membrane anchor (syn1A11) was phosphorylated in vitro with purified CKII. Five hundred nanograms of unphosphorylated (lanes 1 and 3) or phosphorylated (lanes 2 and 4 ) syn1A11 were resolved by SDS-PAGE, transferred to nitrocellulose, and probed with the antibody $\alpha$ Psyn (lanes 1 and 2) or $\alpha$ HPC -1 (lanes 3 and 4 ). Although $\alpha \mathrm{HPC}-1$ equally recognized the phosphorylated and nonphosphorylated syn1A11, $\alpha$ Psyn detected only the phosphorylated protein.

Based on the specificity of the $\alpha$ Psyn antibody, and on the molecular weight and the fractionation behavior of the recognized proteins, we conclude that both phosphosyntaxin $1 \mathrm{~A}$ and $1 \mathrm{~B}$ (p-syn1A, p-syn1B, respectively) are present in the adult rat brain. Figure $2 B$ shows the close sequence similarity of syn $1 A$ and syn1B over the length of the peptide that was used as immunogen. Syn1A and syn1B are closely related isoforms with a high level of sequence homology ( $84 \%$ identity) (Bennett et al., 1993a). The two syntaxin isoforms are expressed in a combinatorial manner in the CNS and PNS, with regions of overlapping and regions of distinct distribution (Ruiz-Montasell et al., 1996; Aguado et al., 1999). The physiological significance of the occurrence of these two close isoforms and their differential distribution remains to be elucidated. The identity of the sequence centered on the
A

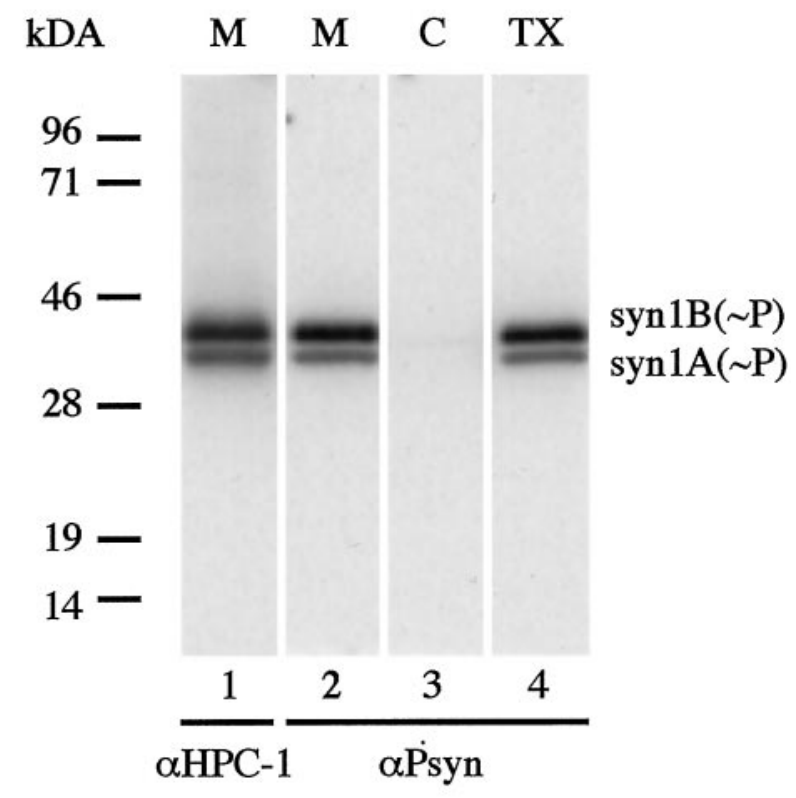

B $\begin{array}{ll}\text { syn1A } & \text { RTAKDS *DDDDD } \\ \text { syn1B } & \text { RSAKDS *DDEEE }\end{array}$

Figure 2. $\alpha$ Psyn recognizes phosphorylated syn1A and syn1B in rat brain fractions. $A$, Rat brain was homogenized and fractionated by differential centrifugation. Total protein $(15 \mu \mathrm{g})$ from the membrane fraction $(M)$, cytosolic fraction $(C)$, and Triton X-100-extracted membrane fraction $(T X)$ was resolved by SDS-PAGE, transferred to nitrocellulose membranes, and probed with $\alpha$ HPC-1 (lane 1) or $\alpha$ Psyn (lanes 2 to 4 ). $\alpha$ HPC- 1 detected a doublet in the membrane fraction; the lower band corresponds to syn1A and the upper band to syn1B. Similarly, $\alpha$ Psyn recognized phosphorylated syn1A and syn1B. As expected, no bands are detected in the cytosolic fraction, and phosphorylated syn1A and syn1B are quantitatively extracted from the membrane fraction by Triton X-100 incubation. $B$, Sequence alignment of syn1A and syn1B over the length of the peptide used as immunogen. Note that although the syn1A peptide was used as immunogen, the sequence of syn1B centered on serine-14 (underlined) is identical to that of syn1A.

phosphoserine (Fig. 2B, underlined) likely explains why the $\alpha$ Psyn antibody, which was raised against the syn1A-derived peptide, also recognizes the phosphorylated syn1B.

We next examined the presence of $\mathrm{p}$-syn1A and $\mathrm{p}$-syn1B in several regions of the rat brain. Homogenates of the different dissected parts of the brain were analyzed by Western blot. The presence of $\mathrm{p}$-syn1A and p-syn1B accurately mirrored that of syn1A and syn1B detected by $\alpha$ HPC -1 (Fig. 3, compare top and bottom panels). Interestingly, some areas of the brain express the two syntaxin 1 isoforms in similar amounts (cortex, hippocampus, and olfactory bulb), whereas other regions show a marked preference for syn1B (cerebellum, medulla, midbrain, and spinal cord). Despite this differential expression of the two isoforms, phosphorylated syntaxin $1 \mathrm{~A}$ and $1 \mathrm{~B}$ are found throughout the whole rat brain.

\section{The extent of phosphorylation on syntaxin 1 is upregulated during development}

After detecting significant levels of p-syn1A and p-syn1B in the brain of adult animals, we investigated the possibility of a devel- 


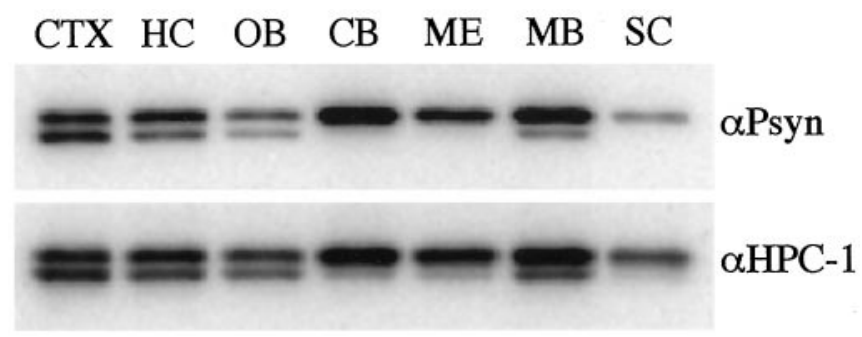

Figure 3. Phosphosyntaxin $1 \mathrm{~A} / \mathrm{B}$ are detected throughout the rat brain. Adult rat brain was dissected in cortex $(C T X)$, hippocampus $(H C)$, olfactory bulb $(O B)$, cerebellum $(C B)$, medulla $(M E)$, midbrain $(M B)$, and spinal cord $(S C)$. The tissues were homogenized, and the postnuclear fraction was prepared for Western blotting. Total protein $(15 \mu \mathrm{g})$ of each tissue was resolved by SDS-PAGE, transferred to nitrocellulose membranes, and probed with $\alpha$ Psyn (top panel) and $\alpha \mathrm{HPC}-1$ (bottom panel).

opmental regulation of the extent of CKII-mediated phosphorylation of syntaxin 1 . To this end, brains of animals of different ages were tested for the presence of p-syn1A and p-syn1B by quantitative Western blot with $\alpha$ Psyn and $\alpha \mathrm{HPC}-1$. For comparison, we also quantified the developmental regulation of expression of two synaptic vesicle (SV) markers, synaptotagmin and VAMP2, and two syntaxin 1-interacting proteins, the soluble factor nsec1 and the plasma membrane protein SNAP-25. A representative Western blot result is shown in Figure 4A. Figure $4 B$ displays its quantitative analysis. For comparison, the amounts of the different proteins were normalized to 100 arbitrary units at the earliest time point, E18. All proteins tested showed increased relative levels of expression during development. The amount of the two SV proteins synaptotagmin and VAMP2 increased steadily, reaching in the adult values of approximately 9- to 12 -fold the amounts detected at E18, a trend that likely reflects the increasing number and maturation of synapses. SNAP-25, nsec1, syn1A, and syn1B (the last two proteins were quantified together and detected with $\alpha \mathrm{HPC}-1$, which recognizes both the phosphorylated and nonphosphorylated forms of the protein) showed a more modest increase in expression, with values in adult that were three- to fourfold above the amounts at E18. Interestingly, the increase in the extent of phosphorylation on syn1A and syn1B (quantified together) paralleled the behavior of the two SV proteins (ninefold increase in adult over E18), substantially differing from the moderate increase in syn1A and syn1B expression. Therefore the proportion of phosphorylated syn1A and syn1B increases with the maturation of the brain.

Using known amounts of in vitro phosphorylated or mockphosphorylated syn1A11 as standards, we calibrated the signal generated by $\alpha$ Psyn and $\alpha$ HPC- 1 in Western blot assays. Using this information we estimated how much of syntaxin 1 is phosphorylated on serine-14 at different time points in development (p-syn1A and p-syn1B were again quantified together). We found that although at E18 only $4 \%$ of syntaxin 1 is phosphorylated, the percentage increased to $17 \%$ at $\mathrm{P} 4$, to $26 \%$ at $\mathrm{P} 12$, and finally reached $40 \%$ in the adult. This high level of phosphorylation on syntaxin 1 is particularly striking if one considers the abundance of this plasma membrane protein in the brain.

\section{Phosphorylated syntaxin 1 is enriched in complexes with SNAP-25}

We next examined the possible regulation of the CKII-mediated phosphorylation of syn1A and syn1B. We prepared acute rat
A

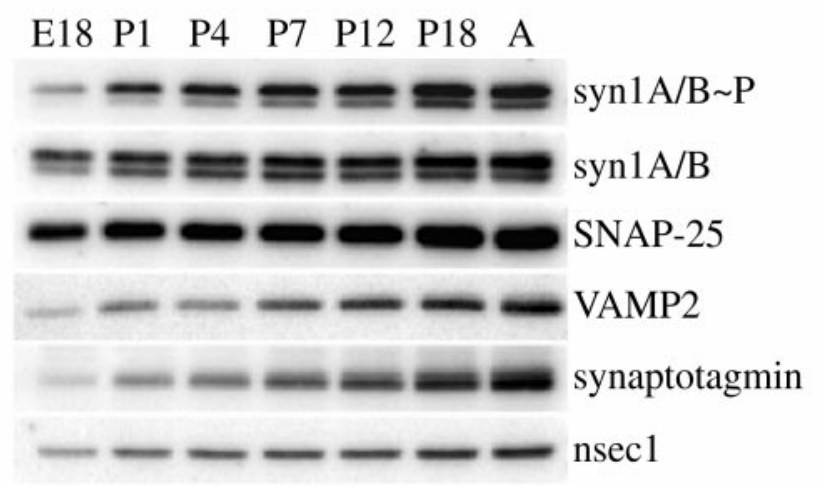

B

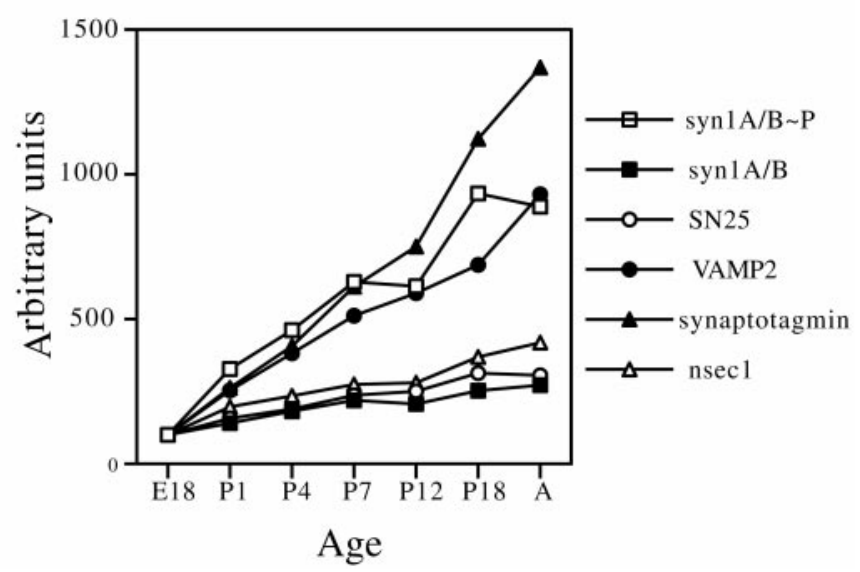

Figure 4. The extent of serine-14-phosphorylation on syn1A/B increases during the development of the rat brain. Rat brains of different ages $(E$, embryonic; $P$, postnatal; $A$, adult) were homogenized, and the postnuclear fraction was extracted with Triton X-100. Total protein $(25 \mu \mathrm{g})$ from each lysate was resolved by SDS-PAGE, transferred to nitrocellulose, and probed with specific antibodies to detect phosphorylated syntaxin1A/B, syn1A/B, SNAP25, VAMP2, synaptotagmin, and nsec1. $A$, Representative Western blot results. $B$, Quantitative analysis of the relative amounts of detected proteins. The data for each protein were normalized to 100 arbitrary units at E18.

brain slices and incubated them under conditions that promote depolarization and SV exocytosis $\left(56 \mathrm{~mm} \mathrm{~K}^{+}\right.$for $1 \mathrm{hr}$ ) or with 50 $\mu \mathrm{M}$ 4-amino-pyridine for $20 \mathrm{~min}$, a treatment that delays action potential repolarization and increases firing rate by selectively blocking the $I_{\mathrm{D}}$ potassium current (Wu and Barish, 1992). We also tested agents known to stimulate CKII (30 $\mu \mathrm{m}$ sphingosine for $1 \mathrm{hr}$ ), PKC (10 $\mu \mathrm{M}$ phorbol 12,13-dibutyrate for $1 \mathrm{hr}$ ) (Fig. $5 A)$, or PKA (50 $\mu \mathrm{M}$ Forskolin for $1 \mathrm{hr}$; data not shown). The extent of phosphorylation of syn1A and syn1B was quantified by Western blot with $\alpha$ Psyn. Comparison with unstimulated slices showed that these treatments failed to alter the state of the serine-14 phosphorylation on syn1A and syn1B (Fig. $5 A$ ). It thus appears that short-term pharmacological stimulations do not have an effect on the serine-14 phosphorylation of syntaxin 1, suggesting that this phosphorylation is unlikely to be involved in any fast change in syntaxin function as a means of modulation in synaptic activity.

The phosphorylation on serine-14 is positioned away from the $\mathrm{H} 3$ domain of syntaxin, the $\mathrm{C}$-terminal $\alpha$-helical region situated 


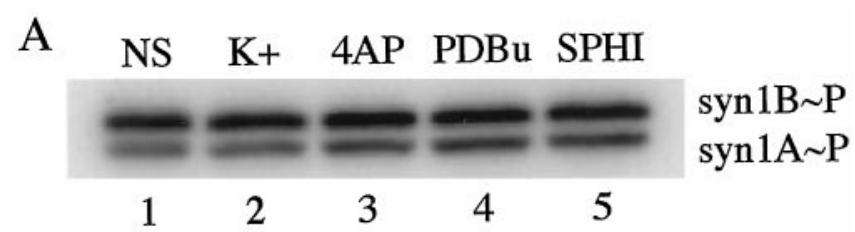

B

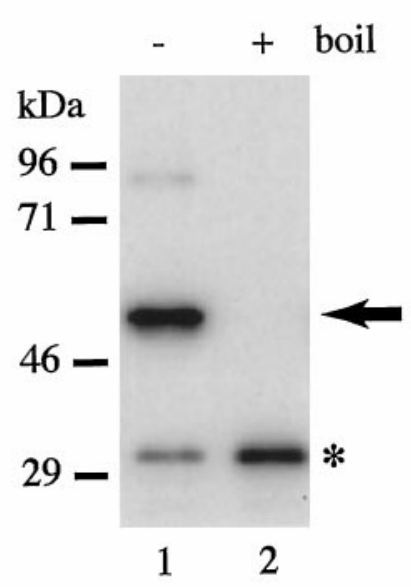

C

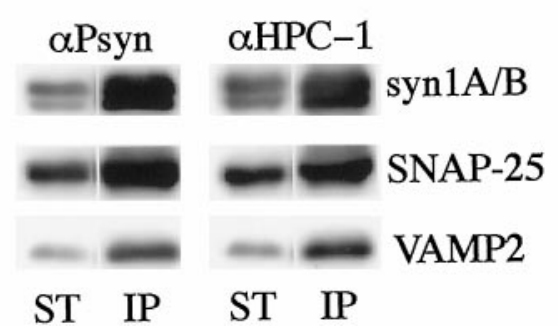

Figure 5. Phosphorylated syntaxin 1 is enriched in complexes with SNAP25. A, Stimulation of acute rat brain slices failed to alter the state of serine-14-phosphorylation on syntaxin1A/B. Acute rat brain slices were prepared from adult animals and subjected to no stimulation $(N S), 1 \mathrm{hr}$ in $56 \mathrm{~mm}$ potassium $(K+), 20 \mathrm{~min}$ in $50 \mu \mathrm{M}$ 4-amino-pyridine $(4 A P), 1 \mathrm{hr}$ in $10 \mu \mathrm{M}$ phorbol 12,13-dibutyrate $(P D B u)$, or $1 \mathrm{hr}$ in $30 \mu \mathrm{M}$ sphingosine $(S P H I)$. The slices were homogenized, and the homogenate was extracted with Triton X-100. Total protein $(50 \mu \mathrm{g})$ of each lysate was resolved by SDS-PAGE, transferred to nitrocellulose membranes, and probed with $\alpha$ Psyn. $B$, In vitro-phosphorylated syn1A11 readily assembles with VAMP2 $\Delta 1-24$, SN25C, and SN25N to form an SDS-resistant/boilingsensitive core complex. Syn1A11 was in vitro-phosphorylated with purified CKII and then mixed with a twofold molar excess of VAMP2 $\Delta 1-24$, SN25C, and SN25N. After a $6 \mathrm{hr}$ incubation, unboiled (lane 1) or boiled (lane 2) samples were resolved by SDS-PAGE, transferred to nitrocellulose membranes, and probed with $\alpha$ Psyn. Under unboiled conditions (lane 1), phosphorylated syn1A11 was found primarily in a $\sim 50 \mathrm{kDa}$ SDS-resistant complex (arrow) with little protein left in the monomeric form (asterisk). After boiling (lane 2), the SDS-resistant complex disassembled, and the phosphorylated syn1A11 was detected exclusively in the monomeric form. $C$, Phosphorylated syntaxin 1A/B coimmunoprecipitates with SNAP-25. Rat brain was homogenized, and the postnuclear fraction was extracted with Triton X-100. The lysate was incubated with a $\alpha$ SNAP-25 antibody followed by protein-G coupled to Sepharose beads. Total protein $(25 \mu \mathrm{g})$ from the starting material (ST) and one-fifth of the total immunoprecipitated material $(I P)$ were loaded on duplicate and resolved by SDS-PAGE, transferred to nitrocellulose membranes, and finally probed to detect the presence of phosphorylated syntaxin 1A/B $(\alpha P s y n)$, total syntaxin $(\alpha H P C$ 1), SNAP-25, and VAMP2.

just before the membrane anchor. This region has been shown to be essential in the formation of the four-helix bundle structure with SNAP-25 and VAMP. We therefore predicted phosphory- lated syntaxin 1 to be unaffected in its ability to interact with the other components of the core complex. To test this prediction we combined in vitro phosphorylated syn1A11 with VAMP2 $\Delta 1-24$, SN25C, and SN25N (the portions of VAMP2 and SNAP-25 that each contribute one helix to the four-helix bundle) under standard conditions to allow the formation of the minimal core complex (Poirier et al., 1998b; Fasshauer et al., 1998). As with the unphosphorylated syn1A11 (data not shown), we observed efficient formation of the SDS-resistant/boiling-sensitive complex that is a hallmark of the formation of the four-helix bundle (Fig. 5B) (Hayashi et al., 1994).

Of the interactions between syntaxin 1 and its partners, that with nsec1 requires a majority of the cytoplasmic portion of syntaxin (Kee et al., 1995). We therefore also tested the possibility that the phosphorylation on serine-14 could alter the affinity of this binding. A glutathione- $S$-transferase-nsec1 fusion protein was immobilized on glutathione beads, and a bead-binding assay was performed with various concentrations of in vitrophosphorylated or mock-phosphorylated syn1A11. The phosphorylated and unphosphorylated syn1A11 bound to nsec1 with essentially the same affinity (data not shown).

To further explore the ability of p-syn1A/B to interact with SNAP-25 and VAMP2, we subjected rat brain lysates to immunoprecipitation with an antibody specific for SNAP-25. The complexes purified with $\alpha$ SNAP-25 contained, together with SNAP25, phosphorylated syntaxin1A/B and VAMP2 (Fig. 5C). Interestingly, $\mathrm{p}$-syn1A/B was enriched in the immunoprecipitated complexes. Quantitation of the recovered proteins showed that the ratio of p-syn1A/B to total syntaxin increased twofold in the immunoprecipitated material relative to the starting material. Similarly, the relative ratio of p-syn1A/B to SNAP-25 was increased 1.8 -fold in the $\alpha$ SNAP-25 immunoprecipitation relative to the starting material, whereas the proportion of total syntaxin 1 to SNAP-25 showed only a 1.1 -fold increase.

\section{Phosphorylated syntaxin 1 is present at nonsynaptic sites along a subset of axons}

To further understand the physiological significance of the phosphorylation on serine-14 of syntaxin 1, we performed immunohistochemistry on sagittal rat brain sections. We focused our analysis on the cortex, the hippocampal region, and the cerebellar cortex.

Figure $6 A$ is a deconvolution reconstruction of serial images taken in the cortex using $\alpha$ Psyn and TOTO-3 as nuclear marker. The staining for $\mathrm{p}$-syn1A/B shows a punctate distribution that appears to outline a selected number of processes. Preincubation of $\alpha$ Psyn with the phosphopeptide used as immunogen (but not with the related nonphosphopeptide) or omission of the primary antibodies in the immunohistochemistry protocol completely abolished the staining (data not shown). To investigate the nature of these processes, we costained rat brain sections with $\alpha$ Psyn and an antibody directed against MAP2 (a dendritic marker), or $\alpha$ Psyn and an antibody that recognizes tau (an axonal marker). As shown in Figure $6 B$, a confocal image taken in the cortex, there is no colocalization between the dendrites and the processes stained by $\alpha$ Psyn. In fact, the psyn1A/B puncta are typically observed in processes that appear to run parallel to or in between the MAP2-containing dendrites (Fig. 6, arrows). With $\alpha$ Psyn we never observed staining of cell bodies, and the total absence of costaining with dendrites indicates that the labeled processes are axons. As expected from the high density of axons in the cortex, the antibody that recognizes tau generated a strong 
Figure 6. Phosphorylated syntaxin is present at nonsynaptic sites along a subset of axons. Except for the image in $A$, which is a deconvoluted reconstruction of serial sections, all of the images were acquired by a single confocal scan from the cortex of sagittal rat brain sections. $A$, Double staining with $\alpha$ Psyn (green) and TOTO-3 (red). p-syn1A/B appears as puncta that outline a subset of processes. $B$, Double staining with $\alpha$ Psyn (green) and an antibody against MAP2 (red, stains the dendrites); note the absence of colocalization. Arrows point to psyn1A/B processes that run parallel to a dendrite. $C$, Double labeling with $\alpha$ Psyn (greenyellow) and an antibody against tau (red) to stain axons. In contrast to the high density of axons stained for tau (single processes cannot be resolved), $\alpha$ Psyn stains only a selected number of them. $D-F$, Double staining with $\alpha$ HPC -1 ( $D$, red) and $\alpha$ Psyn (E, green); the merged image in $F$ illustrates that psyn1A/B is present in a subset of axons. $G-I$, Double staining with an antibody against SV2A $(G, r e d)$ and $\alpha \operatorname{Psyn}(H$, green $)$; the merged image of $I$ shows no colocalization. $A r$ rows point to a representative area in which the SV2A and psyn1A/B puncta appear mutually exclusive. The pia is up in the direction of the arrowhead. Scale bars: $A, 5 \mu \mathrm{m} ; B, C, 10 \mu \mathrm{m} ; D-F, 50 \mu \mathrm{m}$; $G, H, 5 \mu \mathrm{m}$.
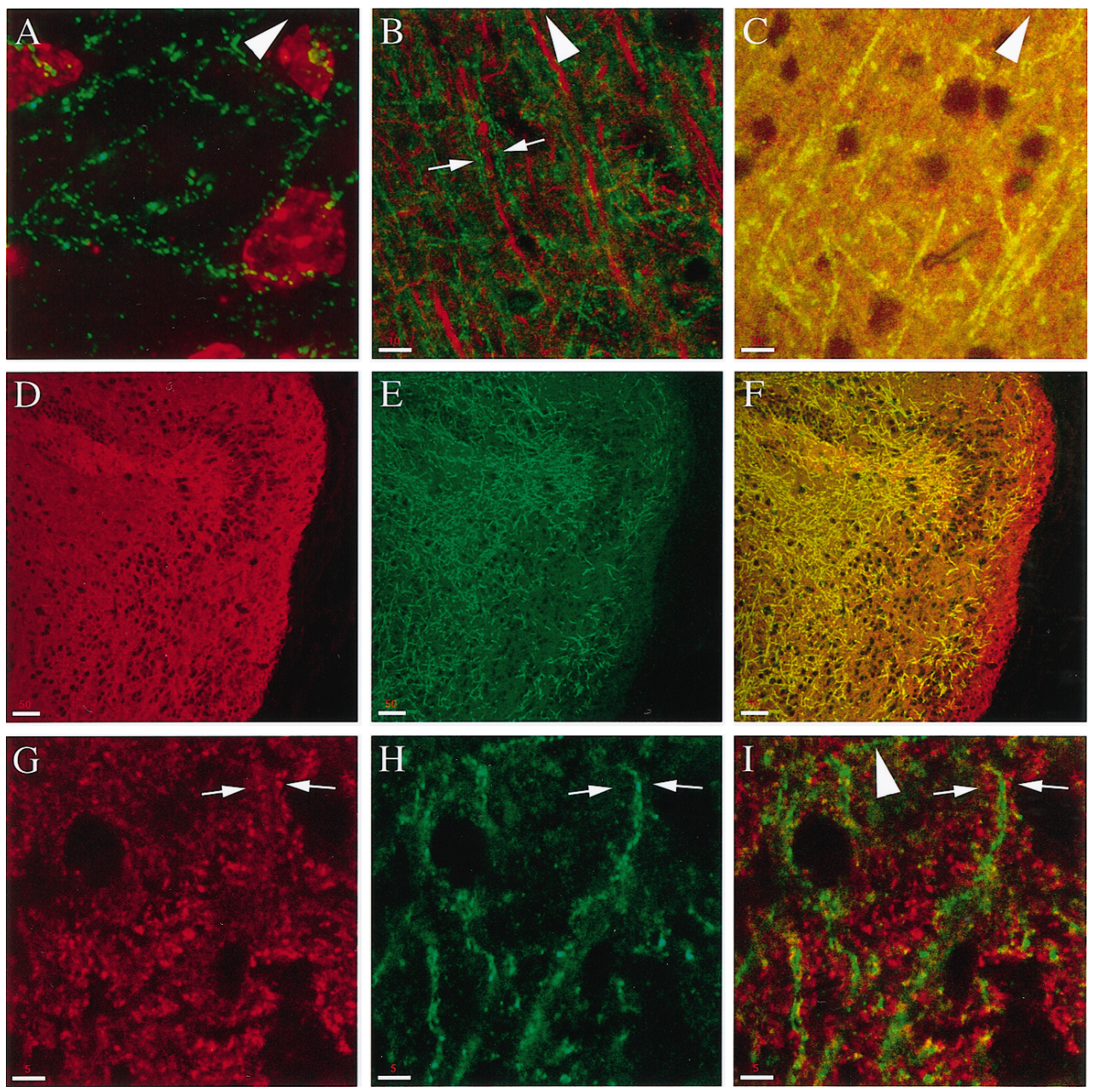

homogeneous labeling, whereas $\alpha$ Psyn stained only a subset of axons (Fig. 6C).

The selective staining of a subset of axons is further illustrated by the costaining with $\alpha$ HPC -1 and $\alpha$ Psyn. $\alpha$ HPC -1 recognizes both the phosphorylated and unphosphorylated form of syntaxin 1. As shown in Figure $6 D$, this results in a homogeneous staining that contrasts with the presence of psyn1A/B in a subset of axons in the same field (Fig. $6 E, F)$. The localization of phosphorylated syntaxin 1 to the axonal domain of neurons is in agreement with previous light and electron microscopy studies (Galli et al., 1995; Garcia et al., 1995; Sesack and Snyder, 1995). Interestingly, the punctate labeling restricted to a subdomain of the plasma membrane is in striking contrast with the homogeneous presence of syntaxin 1 (detected by nonphosphospecific antibodies) along the entire axonal plasmalemma.

The size and distribution of the psyn1A/B puncta were highly reminiscent of staining of synapses, but when we double-labeled sections with $\alpha$ Psyn and with antibodies to detect SV pools at synapses [antibodies against SV2a (Fig. 6G-I) or antibodies against synaptophysin; data not shown for the cerebral cortex but see Fig. 7, $C$ and $F$, for double labeling in the cerebellar cortex], we did not detect any colocalization between SV markers and psyn1A/B. In fact, the psyn1A/B and synaptic puncta appeared to be mutually exclusive (Fig. 6G-I, arrows).

In summary, these double-labeling experiments show that psyn1A/B is detected in a selected population of axons with a punctate staining pattern that does not coincide with synapses.

\section{$\alpha$ Psyn strongly labels the molecular layer in the cerebellar cortex}

Because our biochemical characterization of the phosphorylation state of syntaxin 1 revealed a developmental regulation, we stained sagittal rat brain sections of animals at P5 and P9 and compared them with adults. Figure 7 shows a series of doublestaining experiments performed on sections of animals of different ages with images acquired in the cerebellar cortex.

In Figure $7 A$ we costained sections from P5 animals with $\alpha$ Psyn and TOTO-3. In the developing cerebellum we observed strong staining with $\alpha$ Psyn in the forming molecular layer $(M L)$, whereas little psyn1A/B was detected in the granular layer $(G L)$ and virtually no staining was detected in the external germinal layer $(E G L)$. During maturation of the cerebellar cortex, the granule cells in the EGL first become bipolar with their growing processes oriented parallel to the pial surface and perpendicular to the Purkinje cell dendrites. These processes, the axons of the granule cells, form the parallel fibers that elongate over the surface of already formed fibers in an ordered stacking process that proceeds from the bottom upward, progressively displacing the EGL. Then each granule cell extends a third process, perpendicular to the parallel fibers, that elongates into the ML. Finally, the cell body migrates along this process to reach its final position in the molecular layer (Altman, 1972a).

The intense, homogeneous staining of the ML suggests that psyn1A/B is present in the parallel fibers, consistent with the 

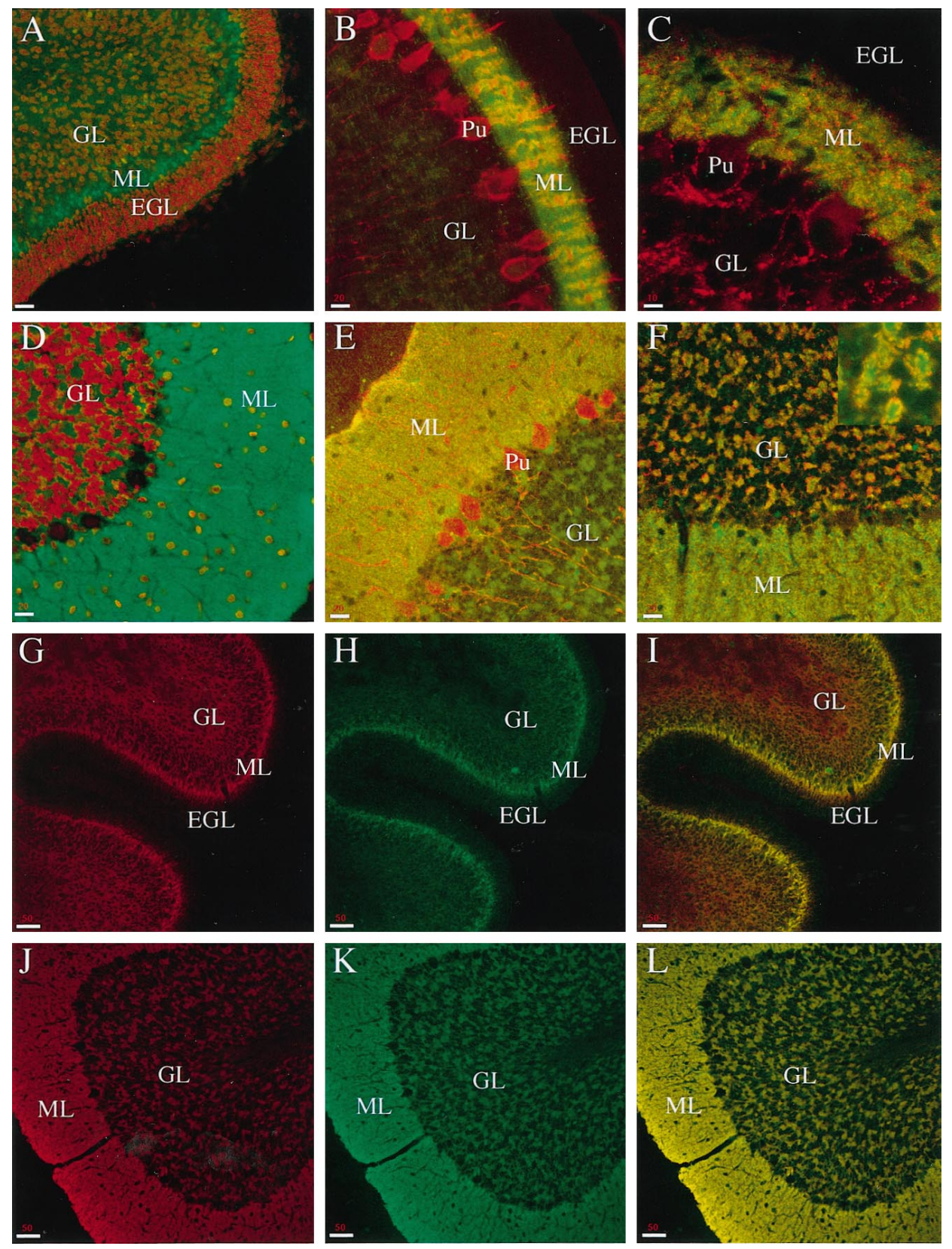

Figure 7. $\alpha$ Psyn strongly labels the molecular layer in the cerebellar cortex. All of the panels represent confocal images taken from sagittal sections of the cerebellar cortex. $A$, Double staining with $\alpha$ Psyn (green) and TOTO-3 (red, stains the nuclei) in P5 animals; psyn1A/B is highly enriched in the forming $M L . B$, Double staining with $\alpha$ Psyn (green) and an antibody against calbindin (red, stains the Purkinje cells) in P9 animals. The maturing Purkinje cell dendrites in the ML appear embedded in $\alpha$ Psyn staining; no psyn1A/B is detected in the EGL, and only low levels are present in the GL. $C$, Double staining with $\alpha$ Psyn (green) and an antibody against synaptophysin (red, stains synaptic vesicles) in P9 animals. No yellow areas of overlap between psyn1A/B and synaptophysin are detected; moreover, the cell bodies (decorated with synapses) and dendrites of Purkinje cells $(P u)$ are not labeled with $\alpha$ Psyn. $D$, Double staining with $\alpha$ Psyn (green) and TOTO-3 (red) in adult animals. The $M L$, still strongly labeled with $\alpha$ Psyn, extends all the way to the pia. Unlabeled Purkinje cell dendrites are apparent, and substantial staining is now observed also in the $M L$ in areas free of cell bodies. $E$, Double staining with $\alpha$ Psyn (green) and an antibody against calbindin (red) in adult animals. $\alpha$ Psyn stains intensely the ML and the GL in discrete structures. The onecell Purkinje cell layer extends highly branched dendrites in the ML and axons across the GL; in both regions there is no colocalization with psyn1A/B. $F$, Double staining with $\alpha$ Psyn (green) and an antibody against synaptophysin (red). The psyn1A/B-positive structures in the GL (inset) are decorated by patches of synapses identifying them as cerebellar glomeruli. As observed in the cortex, the $\alpha$ Psyn staining does not overlap with that of synapses. $G-I$, Double staining with $\alpha$ HPC $-1(G)$ and $\alpha$ Psyn $(H)$ in P5 animals. Although psyn $1 \mathrm{~A} / \mathrm{B}$ is present mainly in the forming ML, $\alpha \mathrm{HPC}-1$ detects the presence of unphosphorylated syntaxin 1 in the GL. I, The merged image with areas of overlap in yellow. $J-L$, Double staining with $\alpha \mathrm{HPC}-1(J)$ and $\alpha \mathrm{Psyn}$ $(K)$ in adult animals. In the adult,

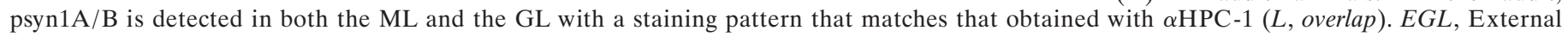
germinal layer; $G L$, granular layer; $M L$, molecular layer; $P u$, Purkinje cell. Scale bars: $A, B, D-F, 20 \mu \mathrm{m} ; C, 10 \mu \mathrm{m} ; G-L, 50 \mu \mathrm{m}$.

axonal distribution observed in the cortex. The parallel fibers are the orderly oriented, tightly packed axons of the granule cells. The high density of staining that results from the structure of the ML is a likely explanation of our inability to detect single puncta as observed in the cortex, where only a subset of axons was labeled.

The preferential staining of the ML is persistent in P9. In Figure $7 B$ we costained with $\alpha$ Psyn and an antibody that specifically labels Purkinje cells (an antibody against calbindin). At this time in development the Purkinje cells have already formed their single cell layer just below the molecular layer, where their dendritic tree is undergoing active growth and branching (Altman, 1972b). As seen at P5, psyn1A/B is detected primarily in the ML at P9, with staining that appears to surround the unlabeled Purkinje cell dendrites; no labeling is observed in the EGL or GL.
In Figure $7 C$ we double-labeled for psyn1A/B and synaptophysin. Confirming the results obtained in the cortex, the psyn1A/B staining is clearly excluded from the areas occupied by the unstained dendrites of the Purkinje cells and does not appear to colocalize with the synaptic puncta scattered throughout the ML. We next performed equivalent costaining experiments in sections from adult rat cerebellum: $\alpha$ Psyn with the nuclear marker TOTO-3, with an antibody against calbindin, and with an antibody against synaptophysin in Figure $7, D, E$, and $F$, respectively. In the adult the EGL has disappeared and the ML extends all the way to the pia. As observed in younger animals, the ML is strongly labeled with $\alpha$ Psyn (Fig. $7 D-F$ ), the Purkinje cell dendrites are not stained (unlabeled ghosts in Fig. $7 D$, no overlap in Fig. $7 E$ ), and there is no apparent colocalization with the SV marker synaptophysin (Fig. $7 F$ ). In contrast to what we observed 
Figure 8. The distribution of phosphorylated syntaxin 1 changes in the cortex and hippocampal region during development. All of the panels represent confocal images of rat brain sagittal sections double-labeled with $\alpha$ Psyn (green) and TOTO-3 (red). A, $B$, In $\mathrm{P} 9$ animals the $\mathrm{CA} 3$ region of the hippocampus shows enriched presence of psyn1A/B. $C$, In the cortex of $\mathrm{P} 9$ animals, the psy1A/B-positive axons are found in the inner layers, but the staining does not extend to the pial surface. $D-F$, In adult animals the cortex shows $\alpha$ Psyn-stained axons throughout its layers $(D, F)$, all the way up to the pia $(E)$. $G-I$, The hippocampus of adult animals is intensely stained with $\alpha$ Psyn $(G)$. In the CA1 region $(H)$, numerous axons are observed in the stratum oriens and stratum radiatum. The dentate gyrus $(I)$ shows intense staining in the hilus and high density of labeled axons in the stratum moleculare. $C A 1, \mathrm{CA} 1$ region of the hippocampus; $C A 3, \mathrm{CA} 3$ region of the hippocampus; $D G$, dentate gyrus; $h i$, hilus; str. m., stratum moleculare; str. o., stratum oriens; str. $r$., stratum radiatum. The pia is up in the direction of the arrowhead. Scale bars: $A, G, 100 \mu \mathrm{m} ; B, D, H, I, 50 \mu \mathrm{m} ; C, E$, $20 \mu \mathrm{m} ; F, 10 \mu \mathrm{m}$.
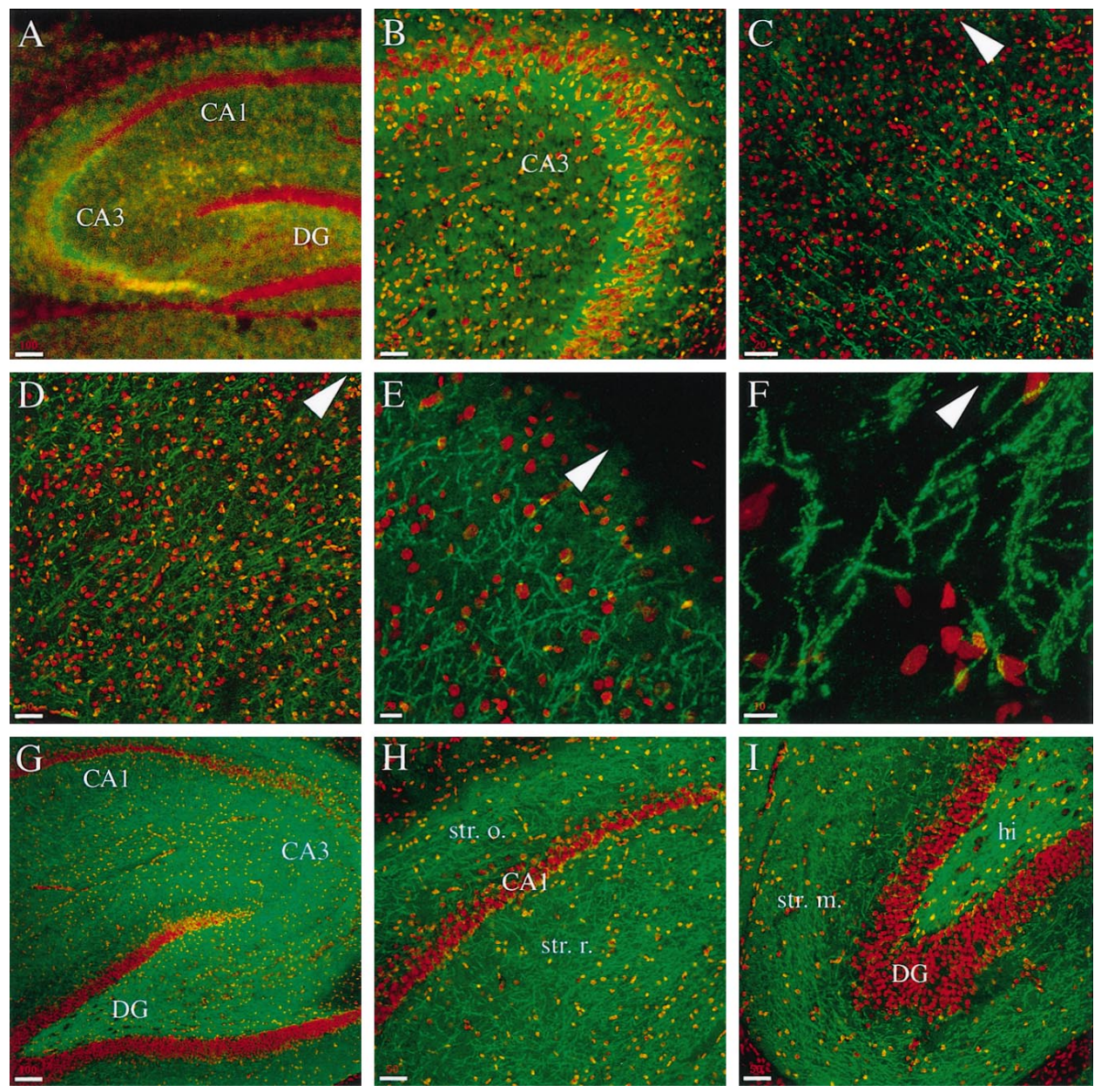

in P5 and P9 animals, we detected significant levels of psyn1A/B in the granular layer. The $\alpha$ Psyn immunoreactivity was confined to areas free of cell bodies (Fig. 7D) that were intensely labeled, on their profile, with the SV marker synaptophysin (Fig. $7 F$ ). The morphology and staining pattern of these structures identify them as the cerebellar glomeruli, the cell-free islands where the mossy fiber rosettes form synapses with the granule cell dendrites, which in turn can be in synaptic contact with the Golgi cell axons (Altman, 1972c). Note again that the synaptic vesicle marker does not overlap with p-syn 1A/B in the glomeruli (Fig. $7 F$, inset).

To further analyze the developmental changes in the distribution of psyn1A/B in the molecular and granular layers of the cerebellum, we costained sections from P5 and adult animals with $\alpha$ HPC -1 and $\alpha$ Psyn. In P5 animals, $\alpha$ HPC -1 stains both the forming ML and the GL; little labeling is detected in the EGL (Fig. $7 G$ ). In contrast, as described above, $\alpha$ Psyn stains primarily the ML (Fig. $7 H$ ), as best shown in the merged image of Figure $7 I$ with the yellow area of overlap restricted to the molecular layer. So, at an early stage in development, while syntaxin 1 is present in neurons in the ML as well as in the GL, psyn1A/B is enriched in the parallel fibers of the molecular layer. In the adult, the distribution of phosphorylated syntaxin 1 becomes more similar to that of unphosphorylated syntaxin 1: $\alpha$ Psyn stains both the ML and the GL (Fig. $7 K$ ) with a pattern and intensity that matches the staining obtained with $\alpha \mathrm{HPC}-1$ (Fig. $7 J, L)$. The distribution and developmental changes that we observed in the rat cerebellar cortex stained with $\alpha \mathrm{HPC}-1$ to detect total syntaxin 1 are in good agreement with previous light and electron micros- copy studies (Koh et al., 1993; Veeranna et al., 1996). Interestingly however, $\alpha$ Psyn revealed that only a specific subset of syntaxin $1 \mathrm{~A} / \mathrm{B}$ is phosphorylated in the developing cerebellar cortex; indeed, the staining was largely restricted to the parallel fibers in the growing molecular layer. In the adult, the distribution of $\mathrm{p}$-syn1A/B became indistinguishable from that of total syntaxin.

\section{The distribution of phosphorylated syntaxin 1 follows the maturation of the cerebral cortex and hippocampal region during development}

We next examined the distribution of psyn1A/B in the hippocampal region and in the cortex, again comparing P9 animals with adults. In the hippocampus of P9 animals we detected intense labeling with $\alpha$ Psyn in the CA3 region (Fig. 8A,B). In the cortex the stained axons, mostly oriented perpendicular to the pial surface, are observed only in the inner layers (Fig. $8 C$ ). In the adult the psyn1A/B-positive axons are detected throughout the cortex (Fig. 8D,F), and labeling is observed up to the pia (Fig. $8 E$ ). The hippocampal region of adult animals displays strong staining with a high density of labeled axons throughout the whole structure (Fig. $8 G$ ). Figure $8 H$ shows a higher magnification of a portion of the CA1 region; psyn1A/B is detected in axons running in the stratum oriens and stratum radiatum, as well as in the stratum lacunosum-moleculare (out of field in Fig. $8 H$ ). A higher magnification of part of the dentate gyrus is shown in Figure $8 I$; a high density of axons containing psyn1A/B is found in the hilus, and numerous processes are stained in the stratum 
moleculare. Occasionally we observed labeled axons crossing the stratum granulosum, spanning the region occupied by the granule cell bodies between the hilus and the stratum moleculare.

Taken together, the immunohistochemical studies support and expand our biochemical data. The staining revealed that of the total pool of syntaxin 1 molecules, only a subset is phosphorylated by CKII. Moreover, although conceivably all neurons express syntaxin 1 , only a subset of them possesses p-syn1A/B. The developmental changes in the distribution of phosphosyntaxin are in agreement with the observed increased proportion of the phosphorylation and are consistent with a role in mature axons. The subcellular localization along the axolemma but outside synapses indicates that this phosphorylation may not have a direct effect on SV exocytosis but could be involved in the distinction between active zones and nonsynaptic portions of the axonal plasma membrane.

\section{DISCUSSION}

We have generated an antibody ( $\alpha$ Psyn) that selectively recognizes the casein kinase II-mediated phosphorylation of serine-14 on syntaxin $1 \mathrm{~A} / \mathrm{B}$. Using this antibody we showed that this phosphorylation occurs in vivo in the rat brain and that the proportion of phosphorylated syntaxin increases during development: from $\sim 4 \%$ in E18 embryos to $\sim 40 \%$ in adult animals. The developmental regulation and the high proportion of phosphorylated syntaxin 1 are noteworthy considering that syntaxin 1 is one of the most abundant membrane proteins in brain. The upregulation in syntaxin 1 phosphorylation paralleled an increase in the levels of SV proteins and is therefore likely to be functionally linked to the increased number and maturation of synapses. When we subjected rat brain slices to treatments that would stimulate synaptic transmission or the activity of different kinases, we did not observe any effect on the phosphorylation state of syntaxin $1 \mathrm{~A} / \mathrm{B}$. The high proportion of phosphorylated syntaxin 1 and the inability of stimulation to change this proportion suggest that the casein kinase II-mediated phosphorylation of syntaxin $1 \mathrm{~A} / \mathrm{B}$ is likely not an activity-induced fast response that could serve rapid modulation of the vesicle docking and fusion machinery. A rapid change in the phosphorylation state after stimulation was observed, for example, in the well characterized case of phosphorylation of synapsin I (Greengard et al., 1993).

Our biochemical comparison between phosphorylated and unphosphorylated syntaxin $1 \mathrm{~A}$ showed that both proteins assembled equally well in the core complex with VAMP2 and SNAP-25, and they bind with similar affinity to the interacting protein nsec-1. Immunoprecipitation experiments confirmed that $\mathrm{p}$-syn1A/B can be found in complexes with SNAP-25 and VAMP2. Interestingly, phosphorylated syntaxin $1 \mathrm{~A} / \mathrm{B}$ was enriched, compared with total syntaxin, in complexes with SNAP-25.

Is phosphosyntaxin $1 \mathrm{~A} / \mathrm{B}$ and its preferential interaction with SNAP-25 marking plasma membrane domains for specific functions, and if so what might that function be? The immunohistochemistry experiments revealed that the $\alpha$ Psyn staining was concentrated on a subset of axons with labeling of puncta that appeared to outline the profile of the processes, consistent with a plasma membrane localization of the phosphorylated syntaxin $1 \mathrm{~A} / \mathrm{B}$. This restricted localization contrasted the widespread distribution along the entire axonal plasma membrane observed for total syntaxin $1 \mathrm{~A} / \mathrm{B}$ stained with $\alpha \mathrm{HPC}-1$. When we doublelabeled sections for $\mathrm{p}$-syn1A/B and SV markers, we did not see any colocalization, strongly suggesting that $\mathrm{p}$-syn1A/B is not enriched at active zones.
The immunohistochemical comparison between young animals and adults confirmed the developmental increase in phosphorylation of syntaxin 1A/B observed by Western blotting as well as the selective distribution of $\mathrm{p}$-syn1A/B staining compared with $\alpha \mathrm{HPC}-1$ staining.

In the cerebellar cortex of young animals, p-syn1A/B was highly enriched in the growing molecular layer, with a staining pattern and progression through development strongly indicative of parallel fiber staining. Only in the adult did we observe $\alpha$ Psyn staining in the granular layer, whereas total syntaxin was detected in this region of the cerebellum throughout development. Similar observations were made in the hippocampus and the cerebral cortex. A highly enriched staining in the CA3 region of the hippocampus in P9 animals was followed by a very strong and widespread staining of axons throughout the whole structure in the adult. In the cortex of young animals, stained axons oriented perpendicular to the pia are observed only in the inner layers, but their presence extends all the way to the pial surface in the mature brain.

In summary, the data are consistent with $\alpha$ Psyn detecting p-syn1A/B preferentially bound to SNAP-25 in binary complexes on the plasma membrane that are outside of synaptic sites. Could this complex be marking plasma membrane domains where exocytosis of specifically synaptic vesicle is inhibited? This interpretation would be consistent with our findings that the proportion of phosphorylated syntaxin $1 \mathrm{~A} / \mathrm{B}$ increases during development and by the progression of the stained axons in both the cerebral and cerebellar cortex. Early in development, flexibility in the choice of the axonal plasma membrane domain that may become a mature presynaptic site is dictated by the transient and developing nature of the synapses. At this time point, a clear and rigid distinction between synaptic and nonsynaptic plasma membrane domains seems not only unnecessary but also possibly detrimental. As axons mature and establish defined synaptic specialization with dendrites, neurons invoke mechanisms to restrict synaptic vesicle exocytosis to the active zones. The presence of phosphorylation on serine-14 of syntaxin could be part of a signaling mechanism to mark the difference between the two domains. Despite the fact that active zones occupy only a small portion of the axonal plasma membrane, several studies have shown that both syntaxin 1 and SNAP-25 are found all along the axolemma (Galli et al., 1995; Garcia et al., 1995).

Alternatively, because phosphosyntaxin is preferentially associated with SNAP-25, it may be that the labeled sites along axons are novel vesicle fusion sites not previously recognized. These axonal domains are not likely to be fusion sites for synaptic vesicles because these organelles do not appear clustered at phosphosyntaxin sites. Perhaps these assembled t-SNARE complexes demarcate fusion sites for a novel class of vesicles important in nonclassical intercellular communication between neurons. Finally, the phosphosyntaxin sites may define axonal domains that mature into new synapses after appropriate stimulation, perhaps by experience.

The phosphorylation site analyzed in this study is in the very $\mathrm{N}$-terminal part of the protein, a location that places it away from the domains that have been shown to be essential in the formation of the four-helix bundle structure that is believed to be at the base of the fusion process of SV with the plasma membrane. Interestingly, among the other plasma membrane syntaxins with broad distribution, syntaxin 3A and syntaxin 4 are also phosphorylated in vitro by CaMKII and CKII, respectively (Risinger and Bennett, 1999). In each case the phosphorylation site was mapped to 
their N-terminal domain. Although this location makes it unlikely that these phosphorylations will have a direct effect on the vesicle fusion process, it indicates that the phosphorylated $\mathrm{N}$ terminus of the protein might have regulatory functions and could interact with other molecules and impart additional functions to syntaxin 1.

\section{REFERENCES}

Aguado F, Majo G, Ruiz-Montasell B, Llorens J, Marsal J, Blasi J (1999) Syntaxin $1 \mathrm{~A}$ and $1 \mathrm{~B}$ display distinct distribution patterns in the rat peripheral nervous system. Neuroscience 88:437-446.

Altman J (1972a) Postnatal development of the cerebellar cortex in the rat. I. The external germinal layer and the transitional molecular layer. J Comp Neurol 145:353-398.

Altman J (1972b) Postnatal development of the cerebellar cortex in the rat. II. Phases in the maturation of Purkinje cells and of the molecular layer. J Comp Neurol 145:399-464.

Altman J (1972c) Postnatal development of the cerebellar cortex in the rat. III. Maturation of the components of the granule layer. J Comp Neurol 145:465-514.

Barnstable CJ, Hofstein R, Akagawa K (1985) A marker of early amacrine cell development in rat retina. Dev Brain Res 20:286-290.

Bennett MK, Calako N, Scheller RH (1992) Syntaxin: a synaptic protein implicated in docking of synaptic vesicles at presynaptic active zones. Science 257:255-259.

Bennett MK, Garcia-Arraras JE, Elferink LA, Petersen K, Fleming AM, Hazuka CD, Scheller RH (1993a) The syntaxin family of vesicular transport receptors. Cell 74:863-873.

Bennett MK, Miller KG, Scheller RH (1993b) Casein kinase II phosphorylates the synaptic vesicle protein p65. J Neurosci 13:1701-1707.

Fasshauer D, Eliason WK, Brünger AT, Jahn R (1998) Identification of a minimal core of the synaptic SNARE complex sufficient for reversible assembly and disassembly. Biochemistry 37:10354-10362.

Galli T, Garcia PE, Mundigl O, Chilcote TJ, De Camilli P (1995) v- and t- SNAREs in neuronal exocytosis: a need for additional components to define sites of release. Neuropharmacology 34:1351-1360.

Garcia PE, McPherson PS, Chilcote TJ, Takei K, De Camilli P (1995) rbSec1A and B colocalize with syntaxin 1 and SNAP-25 throughout the axon, but are not in a stable complex with syntaxin. J Cell Biol 129:105-120.

Greengard P, Valtorta F, Czernik AJ, Benfanti F (1993) Synaptic vesicle phosphoproteins and regulation of synaptic function. Science 259:780-785.

Hanson PI, Roth R, Morisaki H, Jahn R, Heuser JE (1997) Structure and conformational changes in NSF and its membrane receptor complexes visualized by quick-freeze/deep-etch electron microscopy. Cell 90:523-535.

Hayashi T, McMahon H, Yamasaki S, Binz T, Hata Y, Südhof TC, Niemann H (1994) Synaptic vesicle membrane fusion complex: action of clostridial neurotoxins on assembly. EMBO J 13:5051-5061.

Hirling H, Scheller RH (1996) Phosphorylation of synaptic vesicle proteins: modulation of the $\alpha$-SNAP interaction with the core complex. Proc Natl Acad Sci USA 93:11945-11949.

Kee Y, Lin RC, Hsu SC, Scheller RH (1995) Distinct domains of syntaxin are required for synaptic vesicle fusion complex formation and dissociation. Neuron 14:991-998.
Koh S, Yamamoto A, Inoue A, Inoue Y, Akagawa K, Kawamura Y, Kawamoto K, Tashiro Y (1993) Immunoelectron microscopic localization of the HPC-1 antigen in rat cerebellum. J Neurocytol 22:995-1005.

Lin RC, Scheller RH (1997) Structural organization of the synaptic exocytosis core complex. Neuron 19:1087-1094.

McQuinston AR, Madison DV (1999) Nicotinic receptor activation excites distinct subtypes of interneurons in the rat hippocampus. J Neurosci 19:2887-2896.

Nielander HB, Onofri F, Valtorta F, Schiavo G Montecucco C, Greengard P, Benfanti F (1995) Phosphorylation of VAMP/synaptobrevin in synaptic vesicles by endogenous protein kinases. J Neurochem 65:1712-1720.

Pevsner J, Hsu SC, Braun JEA, Calakos N, Ting AE, Bennett MK, Scheller RH (1994) Specificity and regulation of a synaptic vesicle docking complex. Neuron 13:353-361.

Poirier MA, Xiao W, Macosko JC, Chan C, Shim YK, Bennett MK (1998a) The synaptic SNARE complex is a parallel four-stranded helical bundle. Nat Struct Biol 5:765-769.

Poirier MA, Hao JC, Malkus PN, Chan C, Moore MF, King DS, Bennett MK (1998b) Protease resistance of syntaxin.SNAP-25.VAMP complexes. Implications for assembly and structure. J Biol Chem 273:11370-11377.

Risinger C, Bennett MK (1999) Differential phosphorylation of syntaxin and synaptosome-associated protein of $25 \mathrm{kDa}$ (SNAP-25) isoforms. J Neurochem 72:614-624.

Ruiz-Montasell B, Aguado F, Majo G, Chapman ER, Canals JM, Marsal J, Blasi J (1996) Differential distribution of syntaxin isoforms 1A and $1 \mathrm{~B}$ in the rat central nervous system. Eur J Neurosci 8:2544-2552.

Sesack SR, Snyder CL (1995) Cellular and subcellular localization of syntaxin-like immunoreactivity in the rat striatum and cortex. Neuroscience 67:993-1007.

Shimazaki Y, Nishiki T, Omori A, Sekiguchi M, Kamata Y, Kozaki S, Takahashi M (1996) Phosphorylation of 25-kDa synaptosomeassociated protein: possible involvement in protein kinase $\mathrm{C}$-mediated regulation of neurotransmitter release. J Biol Chem 271:14548-14553.

Steegmaier M, Klumperman J, Foletti DL, Yoo JS, Scheller RH (1999) Vesicle-associated membrane protein 4 is implicated in trans-Golgi network vesicle trafficking. Mol Biol Cell 10:1957-1972.

Südhof T (1995) The synaptic vesicle cycle: a cascade of protein-protein interactions. Nature 375:645-653.

Sutton RB, Fasshauer D, Jahn R, Brünger AT (1998) Crystal structure of a SNARE complex involved in synaptic exocytosis at $2.4 \AA$ resolution. Nature 395:347-353.

Veeranna, Grant P, Pant HC (1996) Expression of p67 (Munc-18), $\mathrm{Cdk} 5, \mathrm{P}-\mathrm{NFH}$ and syntaxin during development of the rat cerebellum. Dev Neurosci 19:172-183.

Wu RL, Barish ME (1992) Two pharmacologically and kinetically distinct transient potassium currents in cultured embryonic mouse hippocampal neurons. J Neurosci 12:2235-2246.

Yang B, Gonzalez L, Prekeris R, Steegmaier M, Advani RJ, Scheller RH (1999) SNARE interactions are not selective: implications for membrane fusion specificity. J Biol Chem 274:5649-5653. 